\title{
Verification of the performance of respiratory synchronisation radiation switchgear by using imaging technique
}

\author{
Mun Kyu Park \\ Tongmyong University, \\ 428, Yongdang-dong, Nam-gu, \\ Busan, South Korea \\ and
}

Korea Institute of Radiological and Medical Sciences,

01812, 75 Nowon-gil, Nowon-gu,

Seoul, South Korea

Email: o429mk@naver.com

\section{Seung Woo Park}

Korea Institute of Radiological and Medical Sciences,

01812, 75 Nowon-gil, Nowon-gu,

Seoul, South Korea

Email: swpark@kirams.re.kr

\section{Jae-Hwan Bae and Dong Hoon Lee*}

Tongmyong University,

428, Yongdang-dong, Nam-gu,

Busan, South Korea

Email: bjhmail@tu.ac.kr

Email:1dh5522@tu.ac.kr

${ }^{*}$ Corresponding author

\begin{abstract}
This study has developed respiratory synchronisation beam switchgear that can perform the real-time gating of treatment beam by synchronising with respiratory signal in order to compensate for the motion of normal tissue or organs which is generated by respiration for radiation applied to tumour in radiotherapy. Respiratory signal obtained by the belt system is applied to the self-developed switchgear motor control program so that the AC servo motor of switchgear can be operated in connection with actual respiration. To verify correct respiratory synchronisation operation, motion data of irradiation area which changes according to respiratory signal is obtained by CCD camera-using imaging technique and compared with original respiratory signal. According to the result, it has been confirmed that the correlation coefficient of two signals is higher than 0.98 , showing that the motion pattern of beam switchgear was very similar to actual respiration.
\end{abstract}

Keywords: beam switchgear; motion control; synchronisation. 
Reference to this paper should be made as follows: Park, M.K., Park, S.W., Bae, J-H. and Lee, D.H. (2016) 'Verification of the performance of respiratory synchronisation radiation switchgear by using imaging technique', Int. J. Advanced Media and Communication, Vol. 6, No. 1, pp.93-101.

Biographical notes: Mun Kyu Park received his BSc in BioMedical Engineering from the TongMyong University of Korea in 2010. He received his MS in Busan TongMyong University in 2015. His research interests include image processing and motion control.

Seung Woo Park received his MS and PhD in Electronic Engineering from Inha University, Incheon, Korea. He has been with the division of medical radiation equipment at Korea Institute of Radiological and Medical Science, Seoul, Korea. His main research interests are in the areas of radiation instrument, radiation image processing and biomedical signal processing.

Jae-Hwan Bae is a Professor in the Department of Game Engineering, Tongmyong University, since 2006. From 1998 to 2006, he is an Assistant Professor in the Department of Computer Games, Tamna University. From 2003 to 2005, he did his PhD in Computer and Communications at the Daegu University. From 1993 to 1998, he worked at Korea Data Communication Co., Ltd.

Dong Hoon Lee received his BS, MS and PhD in the Department of Electronics Engineering from Inha University, Korea in 1987, 1993 and 2001, respectively. $\mathrm{He}$ is a Member of Korean Society of Medical and Biological Engineering, and he is also a Member of RESKO and has served as an Editor, since 2008. His main research area is biological signal measurement and processing for the diagnosis, and also HMI and automation system.

This paper is a revised and expanded version of a paper entitled 'The development of beam switchgear and performance verification for respiratory synchronization' presented at 2015 International Conference on Platform Technology and Service (PlatCon-15), Jeju in South Korea, 26 January, 2015.

\section{Introduction}

In radiotherapy, motion of a target organ or tumour may cause the malposition of the target organ which is different from that of the treatment plan and this may result in inconsistency in dosage distribution. About $7-15 \%$ of radiation dosage change during radiotherapy may significantly decrease local tumour treatment efficiency and increase complications of normal cells (Goitein and Busse, 1975; Dutreix, 1984; Mah et al., 1987). The motion of an organ or tumour may be generated by respiration and heartbeat. Although motion by heartbeat has small displacement, motion by respiration has influence on diaphragm and most of organs below it and shows large displacement. It is reported that the motion of diaphragm shows up to $100 \mathrm{~mm}$ of displacement in case of deep inhalation (Langen and Jones, 2001). Therefore, motion by respiration has to be compensated to protect normal tissue or organs from high-dose radiation applied to tumour for the application of radiotherapy or radiological operation to a body part with large motion such as lung cancer and liver cancer (Ozhasoglu et al., 2008). 
A typical method is respiration gating radiotherapy where irradiation is performed in a certain status during respiratory cycle of a patient (Kubo and Hill, 1996; Li et al., 2006). The ultimate aim of radiotherapy which considers respiratory motion is to reduce displacement error generated by respiratory motion by synchronising irradiation with respiratory signal. Therefore, this study aims to develop respiratory synchronisation beam switchgear that can perform the real-time gating of treatment beam by synchronising with respiratory signal and its control system and verify correct respiration linked operation of the device through the comparison with data obtained by imaging technique prior to clinical application to the dynamic lesion tracking radiotherapy system.

\section{Materials and methods}

\subsection{Respiratory synchronisation beam switchgear control system}

If electrical respiratory signal data obtained by the belt system is input to the beam switchgear motor control program which has been self-developed by using LabView 2012 (National Instruments, USA), it passes through the AC servo motor controller and operates the mechanical respiratory motion model motor according to the voltage which changes in time so that the radiation beam switchgear can be operated in connection with the actual respiratory signal. Figure 1 shows the schematic diagram of the respiratory synchronisation beam switchgear control system.

Figure 1 Schematic diagram of respiratory synchronisation beam switchgear control system (see online version for colours)

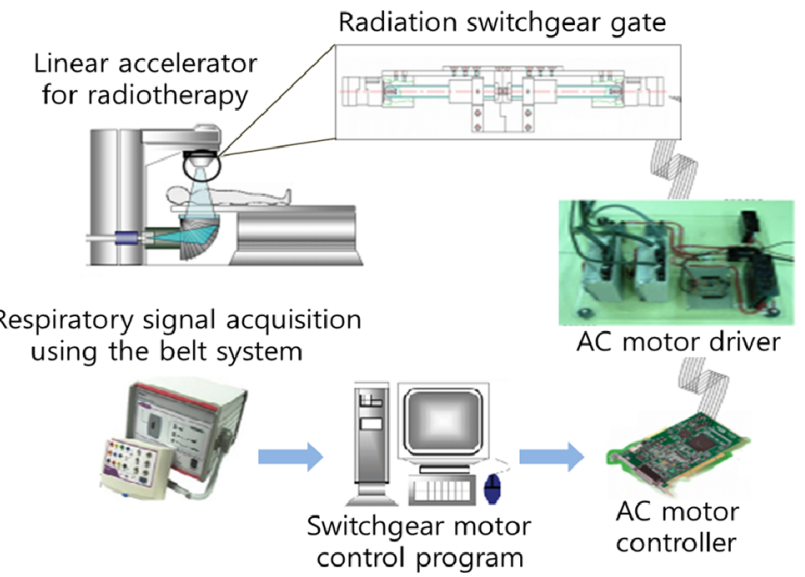

\subsubsection{Respiratory signal acquisition method}

Respiratory signal can be obtained by using various methods. There are typical methods such as locating the target around diaphragm and measuring up/down motion by using the camera, wearing a belt system which uses the strain gauge sensor and measuring the expansion of abdomen during respiration as electrical signal, and measuring respiratory volume by using the lung capacity sensor (Ali et al., 2007; Zhang et al., 2003). This study uses the most common and simple measurement-capable belt system. Poly G-I (LAXTHA Inc., Korea) is used as the instrumentation device. A belt-type respiration 
sensor (RESP Sensor, LAXTHA Inc., Korea) is worn over epigastrium and expansion by respiration is obtained as electrical signal and transmitted to a computer through a USB cable. It is possible to monitor in real time, collect and store data by using a software interface, TeleScan (LAXTHA Inc., Korea). Figure 2 shows respiratory signal acquisition method by using belt system.

Figure 2 Respiratory signal acquisition method by using belt system (see online version for colours)

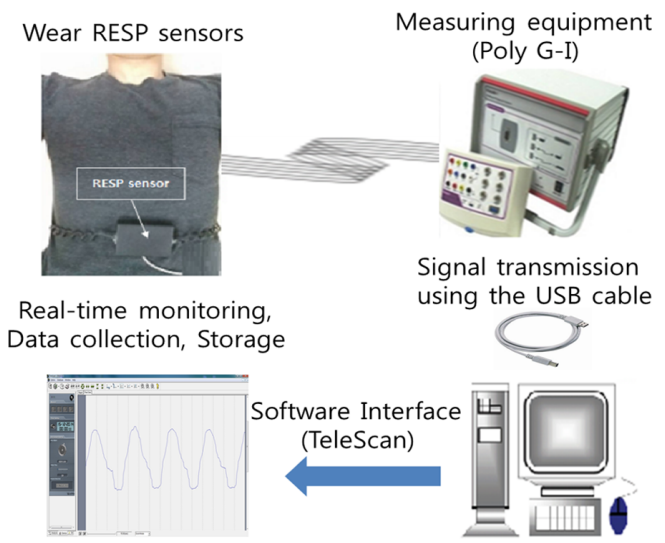

\subsubsection{Making of beam switchgear}

Figure 3 shows the beam switchgear which can perform the gating of treatment beam during radiotherapy. Generally, $6-15 \mathrm{MeV}$ is often used in the clinical field and this study uses tungsten $11 \times 11 \times 4.5 \mathrm{~cm}^{3}$ divided into two parts as the gate in order to select the shielding material which can shield this level as the gate material. Tungsten of $4.5 \mathrm{~cm}$ thickness has radiation transmission rates of $<2 \%, 1.3 \%$ and $0.8 \%$ at $6 \mathrm{MeV}, 10 \mathrm{MeV}$ and $15 \mathrm{MeV}$, respectively. These figures consider scattering effect and can be accepted as clinically shielded (Kim, 2005). The weight of the manufactured tungsten gate is $10,508.85 \mathrm{~g}$ as its density is $19.3 \mathrm{~g} / \mathrm{cm}^{3}$. As it is heavy compared with small volume, aluminium alloy has been selected as the gate frame to ensure low deformation during use. The shielding gate has to switch on/off within $<0.2 \mathrm{~s}$ to ensure clinical effect later. To meet this requirement, $100 \mathrm{~W}$ AC servo motor has been selected to easily control the shielding material of $5254.425 \mathrm{~g}$ which is the weight of one gate.

Figure 3 Self-developed radiation shielding gate (see online version for colours)

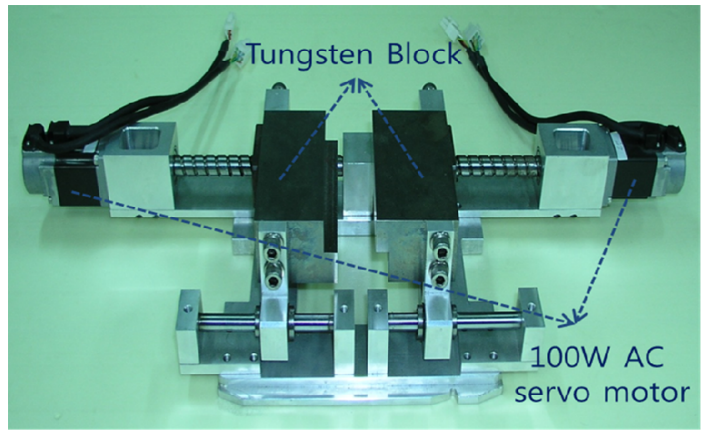




\subsection{Detection of beam switchgear motion by using imaging technique}

CCD camera (BCS-784A, Samsung, Korea) has been used as the device to obtain the motion of beam switchgear which changes in real time in connection with respiratory signal. For image processing, NI Vision Assistant (National Instruments, USA) has been used which provides fundamental functions required for image processing in addition to image acquisition and display to ensure that developers can create a program easily.

First, colour plane extraction function has been used to convert initially obtained colour images to monochromic images and the reference template has been set. Region of interest (ROI) function for specifying analysis area has been used to obtain the information of the desired part only and an imaging technique that utilises the pattern matching function has been used to find an area with an image similar to that of the reference template within the specified area. Next, a user interface has been made by using above image processing functions of Vision Assistant in LabVIEW2012 in order to verify the motion detection of the beam switchgear in real time and collect $y$ values of pattern matching results and saving it in a text file. Figure 4 shows a block diagram which indicates a step-by-step method for detecting the motion of beam switchgear.

Figure 4 Detection of beam switchgear motion by using imaging technique

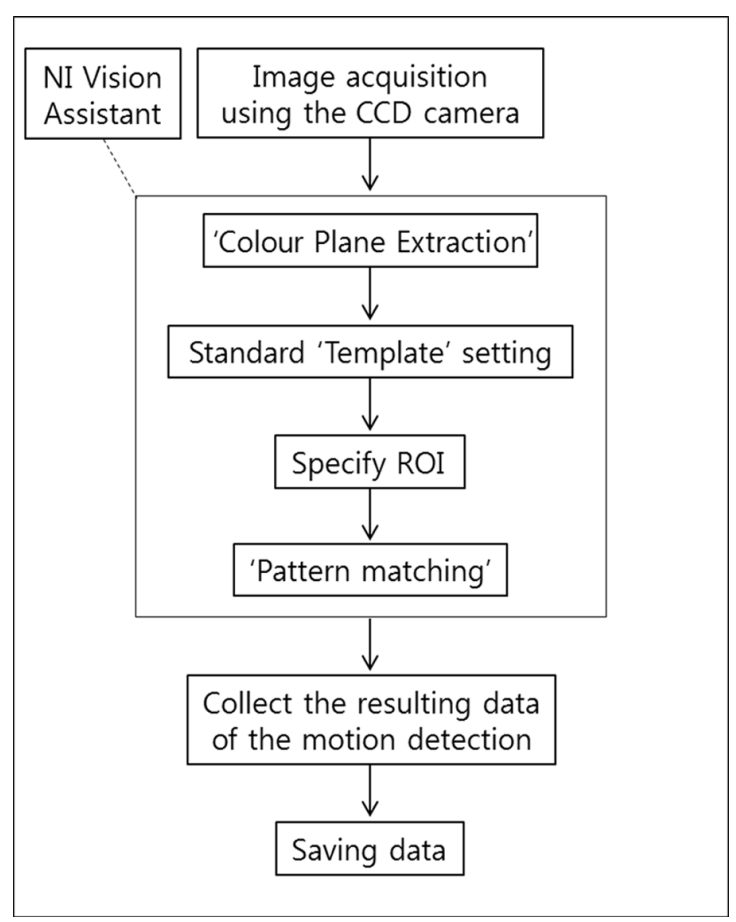

\section{Experiment}

Figure 5 shows the experimental picture to detect the motion of beam switchgear by using imaging technique. The beam switchgear has been installed to the treatment device 
Co-60 (Teratron 780, Canada) in order to verify its correct respiratory synchronisation operation. For safety purpose, light was radiated instead of radiation to indicate irradiation area for simulation. The standard template in the experiment has been set as the irradiation area which is shielded by beam switchgear. Electrical respiratory signal data obtained for $1 \mathrm{~min}$ is input to the beam switchgear control program, the respiration switchgear is operated by the drive signal, and motion signal data of the irradiation area has been obtained by imaging technique using the CCD camera.

Figure 5 Experiment for detecting the motion of beam switchgear by using imaging technique (see online version for colours)

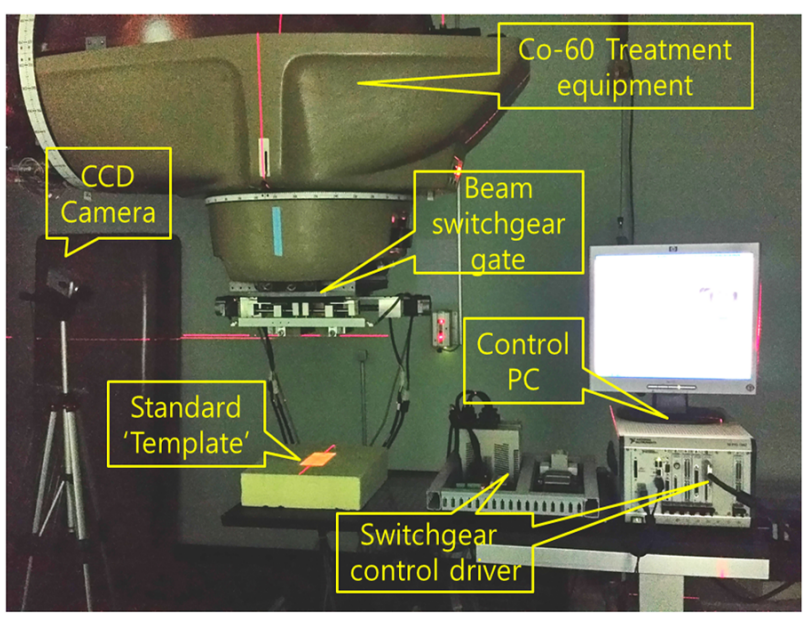

\section{Result and analysis}

\section{4-1 Correlation}

Figure 6 shows the correlation analysis result of human respiratory signal data obtained by the belt system and motion data of the beam switchgear synchronised with the respiratory signal. Correlation analysis is a method for measuring and analysing the degree of linear relation which exists between variables and the correlation coefficient is used as the measure of linear relation. From the graph in Figure 6, the horizontal axis indicates the input signal, respiratory signal data and the vertical axis indicates the output signal, motion signal of beam switchgear measured by imaging technique. First, two signal data were normalised to perform data comparison and the correlation coefficient was applied to the signals as in equation (1) where the respiratory and motion signals have been designated as bivariate variables $\left(x_{i}, y_{i}\right)$.

$$
r=\frac{\sum_{i=1}^{n}\left(x_{i}-\bar{x}\right)\left(y_{i}-\bar{y}\right)}{\sqrt{\sum_{i=1}^{n}\left(x_{i}-\bar{x}\right)^{2}} \sqrt{\sum_{i=1}^{n}\left(y_{i}-\bar{y}\right)^{2}}} .
$$

In the correlation coefficient analysis where more linear relation means closer correlation, the coefficient between the respiratory signal and motion signal has shown 0.986, indicating positive correlation. As shown in Table 1, Richard (1990) has determined that 
when judging from the correlation coefficient, $0.68-1.0$ indicates close correlation and particularly $0.9-1.0$ indicates very close correlation. Therefore, it can be said that patterns of the two signal data show very close correlation according to the experimental result and the developed beam switchgear has excellent operating performance in connection with the input signal.

Table 1 General judgement standard of correlation coefficient

\begin{tabular}{lc}
\hline Correlation coefficient & Analysis \\
\hline $0.90 \sim 1.00$ & Very high \\
$0.68 \sim 0.89$ & High \\
$0.36 \sim 0.67$ & Low \\
$0.00 \sim 0.35$ & Very low \\
\hline
\end{tabular}

Figure 6 Correlation between respiratory and motion signal (see online version for colours)

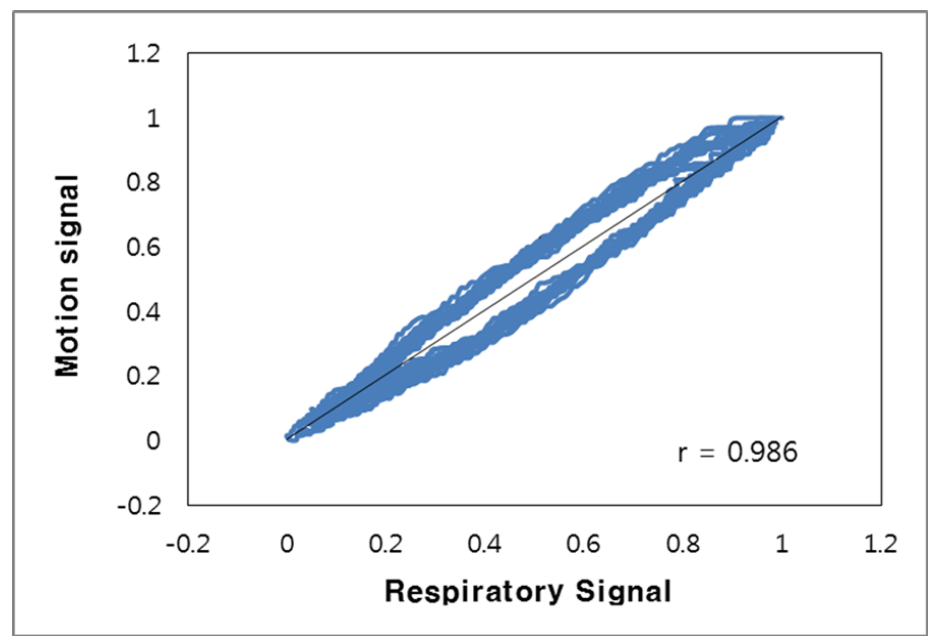

Figure 7 Time delay graph by using LabVIEW 2012 which indicates difference between two signal patterns (see online version for colours)

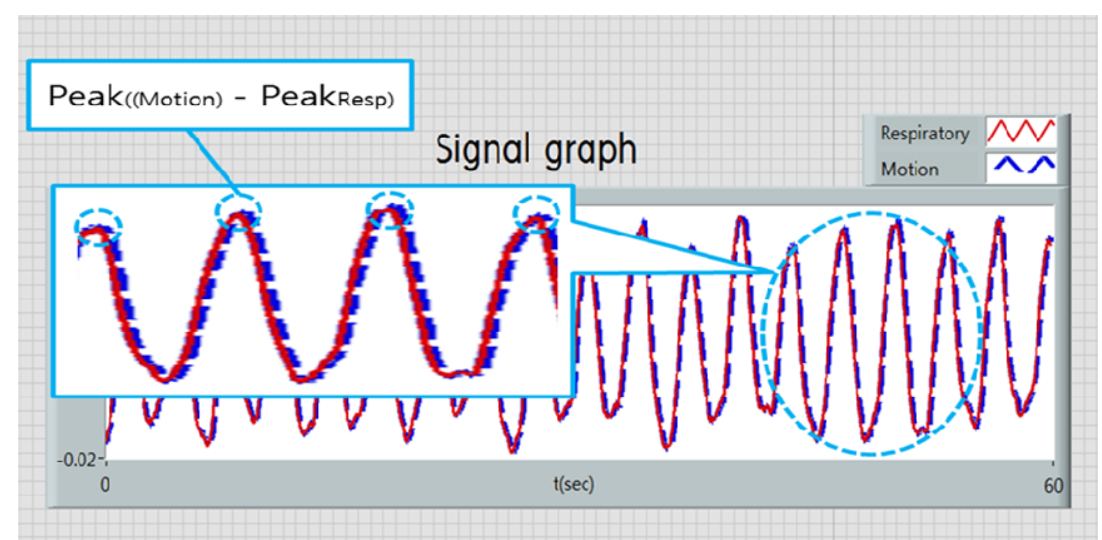




\subsection{Time delay}

According to the comparison of normalised respiratory signal and motion signal data in a graph where the $X$-axis is set as a time variable by using LabView2012, it has been found that as shown in Figure 7, the pattern of the motion signal data has been delayed generally if compared with the respiratory signal pattern. As the code for implementing beam switchgear motor control and imaging technique has been constructed as one program in order to create the interface, it has been confirmed that this is not due to program delay but due to mechanical time delay after the drive signal has been sent. Time data which corresponds to the peak values of respiratory and motion signal data has been detected, and it has been found that $0.095 \mathrm{~s}$ of time delay has occurred according to the averaged difference between peak values of motion and respiratory signals.

\section{Conclusion}

This study intends to develop respiratory synchronisation beam switchgear which can perform the real-time gating of treatment beam by synchronising with respiratory motion and verify its performance through a simple experiment. Actual respiratory signal data were applied to implementation, motion data of irradiation area has been obtained by imaging technique and correlation with original respiratory signal data has been analysed. According to the result, it has been found that the correlation coefficient of two signals was 0.986 , which indicates very close relation and mechanical time delay was $0.095 \mathrm{~s}$. Therefore, it can be judged that the motion operability for pattern of the beam switchgear developed by this study has excellent performance which corresponds to its purpose. It is also judged that if the device is used for clinical application of the dynamic lesion tracking radiotherapy system, it will contribute to the compensation of motion by respiration during treatment and improvement of treatment accuracy, resulting in better clinical effect.

\section{References}

Ali, I., Lovelock, D., Kang, H., Yorke, E., Yamada, Y. and Amols, H. (2007) 'Extraction of internal and external marker 3D-motion in liver patients with compression belt using $\mathrm{KV}$ cone-beam radiographic projections', Med. Phys., Vol. 34, pp.2392.

Dutreix, A. (1984) 'When and how can we improve precision in radiotherapy?', Radiother Oncol., Vol. 2, pp.275-292.

Goitein, M. and Busse, J. (1975) 'Immobilization errors: some theoretical considerations', Radiology, Vol. 117, pp.407-412.

Kim, Y.J. (2005) The Development of Real-time Position Management and Verification System for Respiratory-Gate Radiotherapy, $\mathrm{PhD}$ Thesis, Inha Univ.

Kubo, H.D. and Hill, B.C. (1996) 'Respiration gated radiotherapy treatment: a technical study', Phys. Med. Biol., Vol. 41, pp.83-91.

Langen, K.M. and Jones, D.T.L. (2001) 'Organ motion and its management', Int. J. Radiat. Oncol. Biol. Phys., pp.265-278.

Li, X.A., Stepaniak, C. and Gore, E. (2006) 'Technical and dosimetric aspects of respiratory gating using a pressure-sensor motion monitoring system', Med. Phys., Vol. 33, pp.145-154. 
Mah, K., Dyk, J.B., Keane, T. and Pooh, P.Y. (1987) 'Acute radiation-induced pulmonary damage: a clinical study on the response to fractionated radiotherapy', Int. J. Radiat. Oncol. Biol. Phys., Vol. 13, pp.179-188.

Ozhasoglu, C., Saw, C.B., Chen, H., Burton, S., Komanduri, K., Yue, N.J., Huq, S.M. and Heron, D.E. (2008) 'Synchrony - cyberknife respiratory compensation technology', Med Dosim, Vol. 33, No. 2, pp.117-123.

Richard, T. (1990) 'Interpretation of the correlation coefficient: a basic review', J. Diagn. Med. Sonogr., Vol. 6, No. 1, pp.35-39.

Zhang, T., Keller, H., O'Brien, M.J., Mackie, T.R. and Paliwal, B. (2003) 'Application of the spirometer in respiratory gated Richard Taylor, interpretation of the correlation radiotherapy', Med. Phys., Vol. 30, pp.3165-3172. 